\title{
Self-controlled Exploitation of Energy Cost saving Potentials by Implementing Distributed Demand Side Management
}

\author{
Friederich Kupzog \\ Institute of Computer Technology \\ Vienna University of Technology \\ Gusshausstrasse 27-29 \\ 1040 Vienna, Austria \\ kupzog@ict.tuwien.ac.at
}

\begin{abstract}
Facing the shortage of energy resources and rising energy costs, it is crucial to increase the efficiency of energy usage. Daily consumption peaks in electrical power grids result in the necessity to maintain overcapacities that are only temporarily used. Although already deregulated, today's power markets still lack incentive for large consumer groups to avoid peak consumption. This paper discusses a distributed and integrated load management infrastructure based on a self-controlled load shifting strategy which aims to reduce peak consumption. Essential for its effective and flexible operation is the proposed communication infrastructure, which will enable a flow of information in addition to the flow of energy in national and international power grids. This lowcost and maintenance-free infrastructure will carry real-time pricing information and allow load management appliances to communicate with each other. As a result, the consumer can take part in the energy business and profit from shifting part of the daily load to off-peak hours.
\end{abstract}

Index Terms - Energy Systems, Automation, Load Management, Demand Side Management, Distribution Automation

\section{INTRODUCTION}

While the global demand in electrical energy is increasing steadily, the upgrading of national and international power grids is progressing slowly due to the high economic risks of establishing new power stations. Especially in today's deregulated and dynamic power markets, large longterm investments are preferably avoided. Moreover, there is currently no throughout satisfactory and comprehensive strategy for meeting future global energy demands.

In this urging context a highly efficient usage of existing resources appears to be self-evident. Yet, large potentials for more efficient energy usage are still unexploited. While efficiency in energy consumption is commonly associated only with achieving the same result using less energy, the actual timing of energy usage is usually less focussed on. The deregulation of electricity markets has lead to a strong variability in electricity prices. Daily load peaks result in high daily stock market price peaks, while most energy

Manuscript received June 07, 2006. This project is carried out and financed within the scope of the Austrian Program on Technologies for Sustainable Development, "Energy Systems of tomorrow" - a cooperation of the Austrian Federal Ministry of Transport, Innovation and Technology and the Austrian Research Promotion Agency (FFG).

1-4244-9701-0/06/\$20.00 @2006 IEEE. consumers, particularly small businesses and private households, operate on "flat" tariffs that bear very little motivation to avoid consumption during peak hours.

Ever since public electricity networks have existed, the generator side has had to cope with changing loads during the day, and it has always been the objective to flatten the daily load profile. The conventional approach to reduce consumption peaks is to persuade large consumers (mainly industry and electrical heating) by means of lower prices to shift their consumption times towards the night hours where the vast number of private households has a consumption low point. Nevertheless, this approach is very "coarse grain" and has never been successful enough to avoid additional generation capacities to be installed to cope with daily peak loads. Although a number of isolated initiatives for demand side management (DSM) or load management (LM) exist, these programs are mostly motivated by stability concerns rather than efficiency improvement and still lack the ability to cope dynamically with short-term load changes. In large networks of individually acting and uncoordinated consumers, many small load peaks can add up very easily to one significant peak that leads to efficiency drawbacks and may cause network stability problems. On the one hand, peak avoidance is a very complex problem that cannot be solved without some kind of coordination among the consumers. On the other hand, many power-consuming processes have a flexible time schedule that would allow active peak avoidance given an adequate integrated communication platform. Such a communication infrastructure for inter-consumer load management must be suited for the purpose of short-term or even real time inter-consumer coordination. An integral communication platform could also allow supplying energy consumers with real-time pricing information, opening the possibility for them to gain profit by adapting their consumption patterns according to short-term price changes. Further, integral communication would help to make the coordination between the rising number of small distributed generation (DG) sites and their loads more effective.

Large potentials for more efficient electrical energy usage in general and for reducing peak consumption in particular exist. By implementing a technical and also legal foundation for the self-controlled exploitation of these potentials, the urgent need for more power generation can be 
eased and the installation of new capacities could be avoided or at least delayed.

\section{RELATED WORK}

Aspects of demand side management and distribution automation (DA) have widely been discussed in academia over the past decades. The economic potential and the technical feasibility of distributed and networked DSM systems in particular have already been demonstrated in the 1990s. Generally, contributions in this field can be distinguished as those that deal with the economic aspects (see e.g. [1], [2], [3], [4]) and those that deal with the technical and infrastructural aspects of DSM (see e.g. [5], [6], [7]). Additionally, a number of research and commercial driven applications of DSM solutions have been implemented, see e.g. [8]. However, these practical implementations of DSM systems are either isolated, non-networked solutions or non-automated, manually controlled or even both.

A recent survey of load control programs in North America [7] reveals that the information flow for load curtailment planning is still very basic. Most of the current DSM implementations are installed in North America with the primarily goal to improve system stability. The common incentive for the participants among the surveyed programs is that the changing short-term electricity market price is forwarded to the end-user (to some extend). Energy consumers are informed about time intervals of high demand and high price in which they are rewarded for a reduction of their load. Actual load reduction or switching is performed manually or semi-automated on notifications given up to 24 hours ahead. The information flow (which can be a telephone call in the simplest case) is centralised and participants act autonomously without any direct communication between the participants themselves. Many programmes have been terminated since they failed to adapt to varying system needs. However, such programs in general, along with a number of other case studies (see e.g. [4], [9]), have demonstrated the benefits in stability and economic terms.

DSM programs require a certain degree of predictability of the energy market development. Li and Flynn closely examine the development in electrical energy prices in a broad worldwide selection of deregulated power markets and study the feasibility of using DSM strategies under those market conditions [2]. They subdivide the observed set of markets into three categories "stable markets", "markets with occasional bad price periods" (some of the surveyed markets in [8] fall into this category) as well as "chaotic markets" and show that the emergence of conventional non-networked DSM systems for loads with complex consumption patterns is very unlikely for markets without comprehensible and consistent power price patterns, such as "chaotic markets". This is due to the fact that conventional DSM strategies rely on dependable statistical models for price levels and periodical price development. For an overview of algorithms for load profile prediction and a new approach using artificial neuronal networks see e.g. [10].

A number of strategies and algorithms have been proposed to overcome problems of inflexibility, to optimise the economic benefit and even to enable DSM to be applied in "chaotic markets", where forecasts of load development are unreliable but load shifting would have an even greater positive influence on the market dynamics. A major characteristic of these approaches is that power generators and consumers participate not only in a power distribution network, but also in an information network. This feature, together with the possibility to automate the workflow on the generator and the consumer side, enables a new quality of operation in DSM systems. Further, a variety of additional goals can be achieved, e.g. remote meter reading, remote fault analysis etc. Fig. 1 shows the basic topology of such a combined power and information network proposed by Vyver and Belmans [7]. The authors demonstrate the need for distributed, networked operation for electrical infrastructure control. A distributed control algorithm is proposed, that features fault-propagation avoidance strategies as well as fault tolerance strategies and aims to maximise the availability. It is shown that by establishing a communication infrastructure based on next-neighbour-communication, overall communication is reduced and central-node bottlenecks that occur in the centralized topologies that are currently installed can be resolved. A detailed discussion about the trade-offs between mesh and star topology in control networks can also be found in [5].

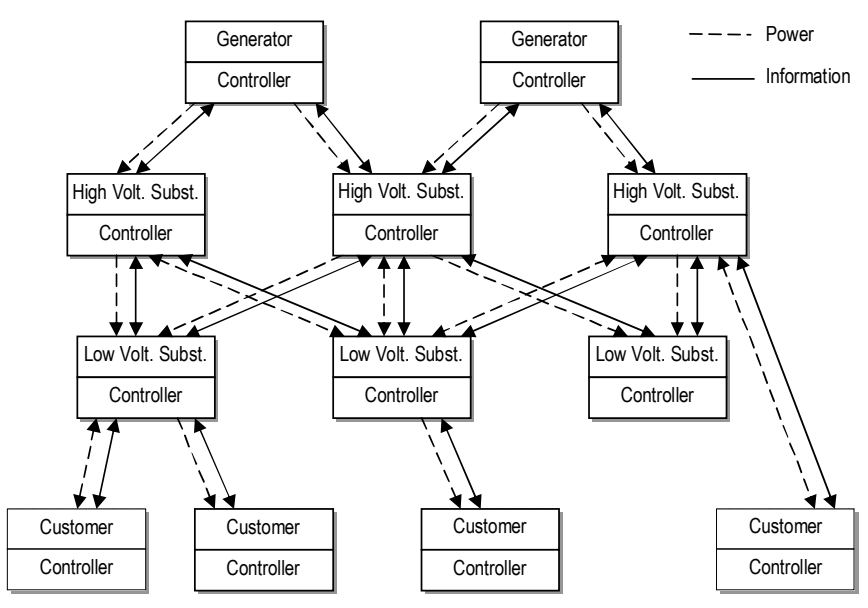

Fig. 1: Example for a combined power and information network proposed by Vyver and Belmans [7].

Other algorithmic proposals - for the still hypothetical case of an adequate communication infrastructure being in place - range from fuzzy set applications [11] to computational agents equipped with artificial intelligence. Ygge, 
Gustavsson and Akkermans [3] model the DSM problem by representing the participants using artificially intelligent agents which act in a computational market economy. Their simulation results reveal that this approach has a very good scalability and achieves cost and energy savings.

In order to advance from currently installed heterogeneous and inflexible solutions towards integrated and potential-exploiting DSM applications, a common communication infrastructure has to be established in parallel to the existing power network. The majority of innovative approaches in this field rely on such an infrastructure and the lack of it causes the large discrepancy between realised DSM applications and the advances in research, which are currently observed.

\section{IRON CONCEPT}

As shown in the previous section, many DSM approaches exist, but the two key features, namely networking and automation, have to be established in order to overcome their inflexibilities and restrictions. Currently installed devices lack these features, which is the main reason for many DSM related products being poorly represented on the market.

A project at the Institute of Computer Technology, Vienna University of Technology, Austria investigates the feasibility, practicability, the algorithms, the way of technical implementation and the large number of related aspects in the interdisciplinary field of distributed DSM. The central idea of this project is to provide a modern information and communication infrastructure for efficient future energy systems: an integrated resource optimisation network (IRON).

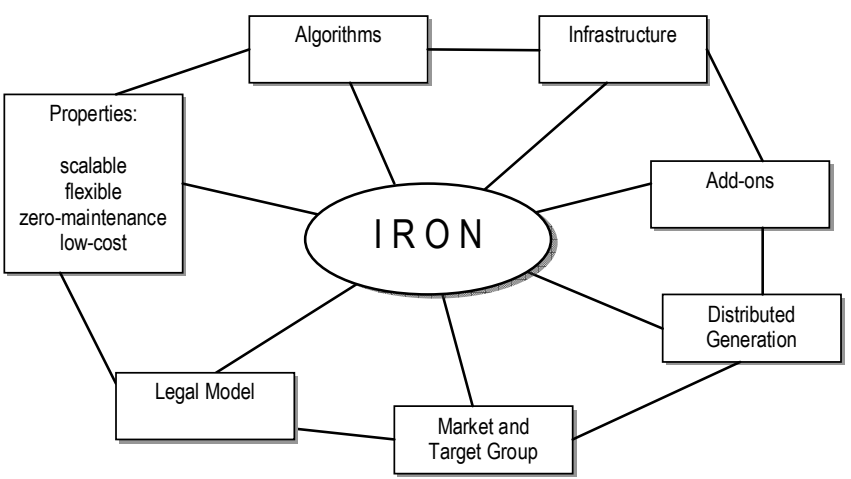

Fig. 2: Overview of the high number of different aspects of the Integrated Resource Optimisation Network (IRON)

Networking is of very high importance for future DSM approaches since peak load reduction can only efficiently be implemented by coordinating multiple loads that are typically geographically distributed. Such a network of dis- tributed consumers is able to achieve a common stable load for a company or region.

Automation is the second key issue, since it enables integration of efficiency-improving hardware into existing installations like industrial plants or private households in such a way that the user is no longer concerned with the operation of the DSM hardware after it has been installed. Only when this feature is available, acceptance for DSM appliances will become broad and reach from the rather small set of large industrial consumers down to the huge number of private households. While the industry is already concerned with questions of DSM due to the larger monetary saving potential in this field, small private consumers predominantly demand simple access to electrical energy and are not willing to trade comfort for small savings. It is important to reach this group though, since it has a large share of total power consumption and therefore also has a strong impact on power economy as a whole.

By providing a common IRON platform for electricity consumers, many novel approaches aiming to improve the efficiency of electrical energy can be practically applied for the first time. For such an integrated solution, which combines expertise in information technology, power technology as well as in topics of economics, a number of different aspects can be identified (see also Fig. 2). In particular, these aspects are:

- Technical infrastructure. The central aim of the IRON project is to specify a common information infrastructure that is able to meet the demands of distributed DSM algorithms and to cope with a vast and growing number of connected entities. The challenge is to identify a suitable and flexible network topology together with a protocol that scales very well and is open for future enhancements. Further, the connection interface between the network and the electrical equipment that is to be load managed has to be specified.

- Algorithms. Once an infrastructure is given, many options for conducting the actual load management exist that have to be investigated in terms of practicability, scalability and efficiency.

- Legal problems. The introduction of an interactive platform that connects not only different technical but also legal entities imposes a number of legal questions that range from ownership, the way profit is shared to security and safety regulations. Current regulations are generally not designed to embed a new and integrated management tool like IRON.

- Economy and market. The implementation of DSM strategies has a number of benefits, but only some of these benefits can be measured in monetary terms. Energy cost savings are determined by the underlying economic model, which defines the incentives for the participants of the market.

- Distributed generation. While current power grids were designed to connect a small number of large power generators with a high number of consumers, 
the increasing exploitation of renewable energies leads to a growing number of small energy generators being connected to different levels of the grid. It has to be investigated how the interaction between distributed generators and consumers could be improved using the IRON platform in order to reduce losses and increase the power quality.

- "Add-on" functionalities, that are not primarily DSMrelated, such as remote meter reading or remote fault analysis.

The IRON infrastructure and algorithms need most constitute a highly flexible and scalable system that is easy to install, virtually maintenance-free and inexpensive. These properties are crucial for the IRON system in order to gain acceptance of the user. Unfortunately, the monetary benefit of load management is cut into a number of pieces that are distributed among the domains of generation, transport and consumption. Consequently, the device and installation costs have to be low, since they are directly reducing the end user's share of the economic benefit.

\section{TECHNICAL INFRASTRUCTURE}

The rapid growth of information technology (IT) related services and equipment over the past decades went along with a strong decline in costs for these products. Hence, the communication system needed for DA and DSM were already considered to be affordable in the 1990s [1]. However, the requirements for such a communication system are very high.

Usually a very large discrepancy between the dependability of power supply and IT equipment is observed. The main reasons for this are the well-established and wellproven power technologies on the one hand and the large number of components and the relatively short lifetime of IT products on the other hand. The IRON communication infrastructure has to keep up with the high standards imposed by the power grid in order to become an accepted and established technology.

Given the possibly large number of single communication nodes that are going to be connected by the IRON communication infrastructure, only a hierarchical network structure appears useful (see also Fig. 3). Due to cost restrictions, the top level (long-distance) infrastructure can only be implemented by using existing communication networks, predominantly the Internet. Different types of sub-entities are then connected to this top-level network. Within these entities, an appropriate sub-network of DSM measurement and controlling units will be present. The size and structure of these sub-networks depend on the kind of entity: for an industrial plant an already present automation infrastructure can be used, or for an office building an already present building automation network can be used. Some entities, such as wind power generators or single remote consumers, are directly connected to the top-level in- frastructure and have no sub-networks.

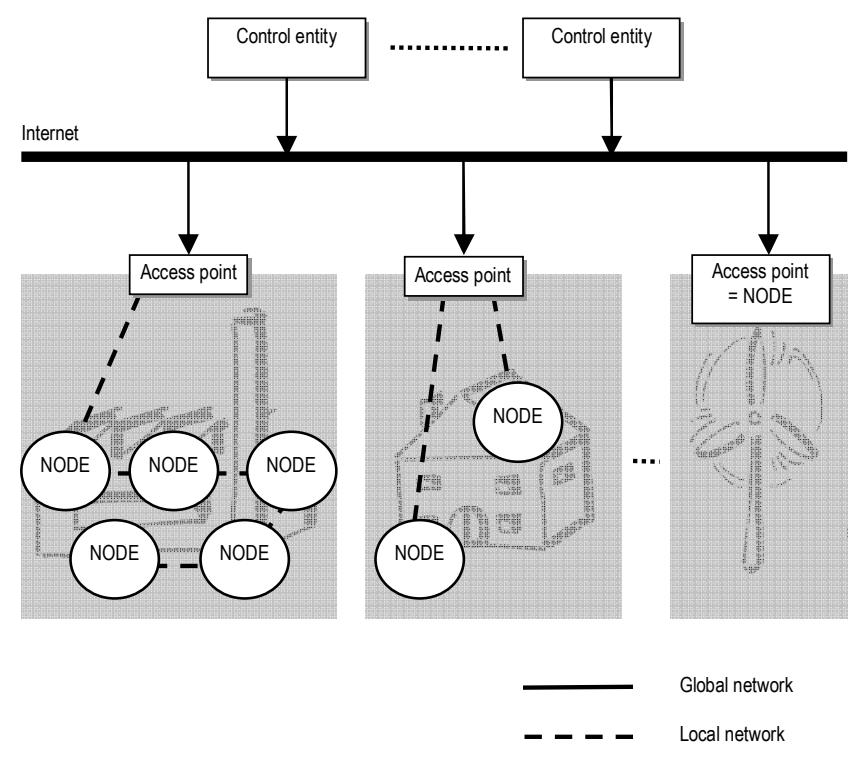

Fig. 3: Top-level and entity-level communication within the IRON infrastructure

Further details on the technical requirements for the IRON infrastructure have already been examined in [12] and [13]. Implementation aspects of using the Internet and public phone networks to establish a distributed data acquisition system, as well as means of improving the dependability of such a system, are discussed in [14].

\section{DSM MODELling AND MARKet SEgMENTATION}

As a result of the deregulation of today's power markets, a relatively large number of interest groups take part in those markets, namely power generation, transport and consumption. The key problem resulting from this fact is that any profit gained by DSM approaches in general and the IRON system in particular has to be shared among these interest groups, making the individual shares rather small. It has been shown [12], that the overall gain of an IRON system on a macro-economic level is positive. However, only parts of it can be measured in monetary terms. It is therefore required to analyse the individual shares in more detail, in order to identify the most appropriate target groups for the IRON system.

As a starting point for this analysis a private household is taken as potential participant, since this is probably the most complicated target for a DSM strategy. The outcome of this first analysis for the private household will show the worst case profit that can be gained from stock market driven DSM. Other target groups, like small businesses or industry, shall be considered in subsequent work, possibly gaining more results from a field trial.

For the estimation of the monetary saving potential of a simple, isolated DSM strategy applied to an average European household, the following simple model is derived (see 
also Table I and Fig. 4). Within this model, the "synthetic load profile" for private households (H0) [15] is used as a representative load profile. Synthetic load profiles are mainly used (and were initiated) by the power supplying industry and serve as reference load profiles for consumers who have no load measurement performed on site. Synthetic load profiles are the result of a statistical analysis based on representative samples.

Table I: Calculating the DSM benefit using real stock market data and a synthetic load profile

\begin{tabular}{|c|c|c|c|c|c|}
\hline Date & Hour & \begin{tabular}{l}
\multicolumn{1}{c}{ Spot } \\
market price \\
(EUR/kWh)
\end{tabular} & $\begin{array}{l}\text { Standard } \\
\text { consump- } \\
\text { tion (kWh) }\end{array}$ & $\begin{array}{l}\text { consump- } \\
\text { tion influ- } \\
\text { enced by } \\
\text { DSM (kWh) }\end{array}$ & $\begin{array}{l}\text { Benefit } \\
\text { (EUR) }\end{array}$ \\
\hline
\end{tabular}

\begin{tabular}{cccccc}
$\vdots$ & $\vdots$ & $\vdots$ & $\vdots$ & $\vdots$ & $\vdots$ \\
\hline $24 / 11 / 2006$ & 16 & 0,13 & 0,11924 & 0,09539 & 0,0032168 \\
\hline $24 / 11 / 2006$ & 17 & 0,27 & 0,14578 & 0,11662 & 0,0079922 \\
\hline $24 / 11 / 2006$ & 18 & 0,24 & 0,1883 & 0,15064 & 0,0090384 \\
\hline $24 / 11 / 2006$ & 19 & 0,13 & 0,21198 & 0,16958 & 0,0055538 \\
\hline $24 / 11 / 2006$ & 20 & 0,08 & 0,19063 & 0,22541 & $-0,0028482$ \\
\hline $24 / 11 / 2006$ & 21 & 0,06 & 0,15975 & 0,19453 & $-0,0022607$ \\
\hline
\end{tabular}

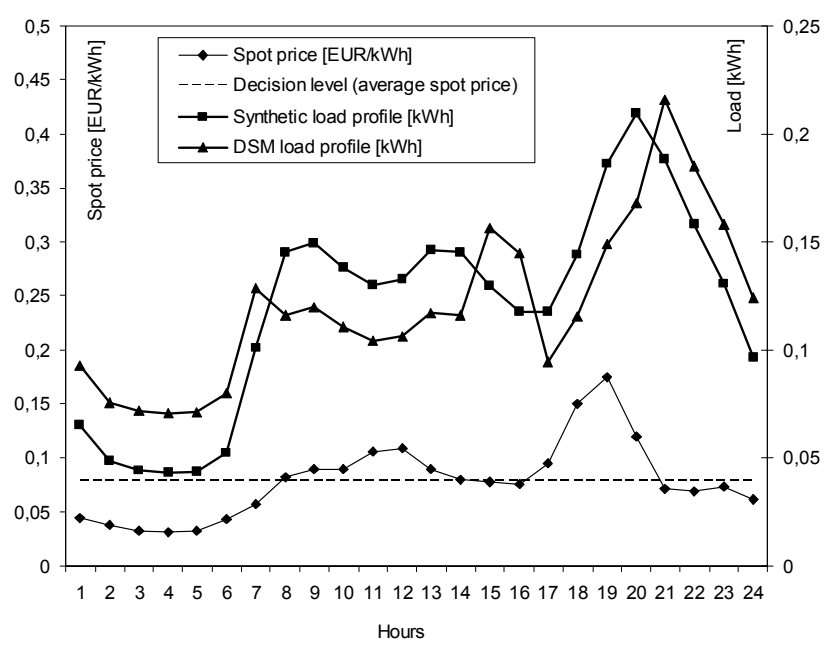

Fig. 4: Stock market price and load profiles for an exemplary day (21/11/2005, European Energy Exchange)

The consumption flexibility of the considered household is modelled by a given fraction of its total daily energy consumption that can be shifted in time. Now, actual stock market prices are taken as a basis to determine daily price peaks. In the model, the considered household reduces its consumption during peak periods, and shifts this consumption to time intervals with lower prices.

The question of whether the current stock market price is considered to be high or low is decided using a critical price level which equals the average price of the examined day (see decision level in Fig. 4). As long as the price is higher than the average value, load reduction is performed.

The calculation, of which a small fraction is shown in
Table I, is performed for a whole year using stock market data of the European Energy Exchange of 2005. For the example of a DSM load potential of $20 \%$, a total energy cost saving of $2.7 \%$ can be achieved.

Although this is a very rough model, it shows the influencing factors for cost saving by DSM strategies and the problems associated with such models very well. First, there has to be a strategy of deciding whether the current electricity price is high or low, or whether a peak or offpeak period is present. The presented model uses the average price as a decision level. In practice, this cannot be implemented since the price curve for a whole day is not known in advance. The decision has to be made taking the price history and predictions into account. Further, the decision can either lead to a discrete "high/low" output or alternatively to a continuous figure.

Further, the actual stock market prices have to be available for the decision to be made. Since the decision for load shifting is performed in real time at the consumer's site (demand side), the price information has to actually be broadcasted to the consumers.

The underlying economic model that enables the consumer to make profit with DSM strategies is crucial. A "flat tariff" does of course not enable any profit. In the model presented, the changes of the stock market price are taken into account and load reduction benefits are forwarded to the consumers. This assumes that the energy supplier is recompensing the consumer's load shift in this way. A number of other economic models are possible, taking into account energy balancing, short-term peak avoidance and other benefits of an IRON infrastructure.

In an attempt to categorise the range of electricity consumers, two main parameters can be identified: first, the total consumption per year and second, the deviation of the load profile. The higher the deviation, the higher the consumer's DSM saving potential in relation to its total energy costs. The higher the consumption, the higher is the saving potential in absolute terms. From the estimation presented above and this segmentation scheme the private households turn out to be the most problematic target group due to their small relative and absolute saving potential. Still, it would be very advantageous in macro-economic terms to reach this group due to its large share in the overall power consumption.

Additionally, the monetary savings are strongly dependant on the "DSM potential" of the considered household, which is the amount of load that can be shifted in time. The estimation presented here assumes that $20 \%$ of the consumer's consumption can be subject to DSM. The actual DSM potential differs with the kind of target group. For private households e.g. heating, warm water and washing machines/dishwashers account to the DSM potential. Small businesses or industry consumers have other, very individual potentials. Another fact, which is not considered in most DSM models including the one discussed here, is that the amount of time the consumption can be delayed is very 
different for various kinds of applications and consumers. This makes it very difficult to precisely estimate the customer behaviour and the economic benefits for a single customer. Reliable figures can only be gained by an actual field trial.

\section{Conclusions ANd Future Work}

The assessment of past and current research activities in the field of distributed DSM on one hand and currently implemented DSM solutions on the other hand reveals a large discrepancy between the theoretically achievable and the actual achieved degree of optimisation of energy usage. The predominant reason for this is the lack of an integrated information infrastructure parallel to the existing power infrastructure, which prevents algorithms and strategies for distributed DSM from being applied. In this paper, the concept for a distributed and integrated resource optimisation network (IRON) is presented, focusing on both the algorithms for distributed DSM and the infrastructure needed. Taking into account the need for scalability and minimum costs, a hierarchical network structure using the Internet for top level communication is proposed.

In order to estimate the economic benefit of DSM applications, a differential model is derived and subsequently used to point out the uncertainties of this kind of estimation. The estimation result of $2.7 \%$ of total energy costs savings for a private household reveals that DSM applications are more likely to be attractive for consumers with a higher yearly consumption such as businesses and industrial consumers. Nevertheless, the outline of hardware appliances that are even attractive for private consumers is still in the central focus of the project, since the large number of private households holds a significant share of total power consumption. In order to gain more realistic results in terms of ecologic and economic saving potentials, the next steps of the project will be to implement a small-scale IRON network and then move on to a field test that will reveal the practical feasibility of the concept.

Future investigation will also focus on an alternative market models. A very promising approach is to interpret a network of several IRON customers as a virtual (negative) power plant, which can be influenced to some extend by the operator of the according control area. This network would therefore be able to take part in the trade of balancing energy and gain profit from this. In contrast to the stock market price model discussed above, where high price periods can last for several hours, the allocation of balance energy usually is only necessary for a fraction of that time. A load shift for e.g. 15 minutes can be achieved much more easily than a shift over several hours.

\section{REFERENCES}

[1] H. L. Smith: DA/DSM directions. An overview of distribution automation and demand-side management with implications of future trends, Computer Applications in Power, IEEE Volume 7, Issue 4, Oct. 1994 , pp. $23-25$

[2] Y. Li, P. C. Flynn, Deregulated Power Prices: Changes Over Time, IEEE Transactions on Power Systems, Volume 20, No. 2, May 2005, pp. $565-572$

[3] F. Ygge, R. Gustavsson, H. Akkermans, HOMEBOTS: Intelligent Agents for Decentralized Load Management, in proc. of Distribution Automation \& Demand Side Management, Vienna, Austria, Oct. 1996

[4] C. Alvarez, A. Gabaldón, A. Molina, Assessment and Simulation of the Responsive Demand Potential in End-User Facilities: Application to a University Customer, IEEE Transactions on Power Systems, Vol. 19, No. 2, May 2004, pp. 1223 - 1231

[5] S. Mak, D. Radford, Communication system requirements for implementation of a large scale demand side management and distribution automation, IEEE Transactions on Power Delivery, Vol. 11 No. 2, Apr. 1996, pp. $683-689$

[6] C. Nunn, Customer communications for demand-side management, IEE Colloquium on Demand-side Management and Resource Planning in the United Kingdom and Europe (Digest No. 1994/186), pp. $3 / 1-3 / 5$

[7] J. v. d. Vver, G. Deconinck, R. Belmans, The Need for a Distributed Algorithm for Control of the Electrical Power Infrastructure, in proc. of the International Symposium on Computational Intelligence for Measurement Systems and Applications, Lugarno, Switzerland, Jul. 2003

[8] P. Jazayeri, A. Schellenberg. W. D. Rosehart, J. Doudna, S. Widergren, D. Lawrence, J. Mickey, S. Jones, A Survey of Load Control Programs for System and Price Stability, IEEE Transactions on Power Systems, Volume 20, Issue 3, Aug. 2005, pp. $1504-1509$

[9] K. Bhattacharyya, M. L. Crow, A Fuzzy Logic Based Approach to Direct Load Control, IEEE Transactions on Power Systems, Vol. 11, No. 2, May 1996, pp- $708-714$

[10] T. Rausch, P. Palensky, PROFESY: Intelligent Global Energy Management, in proc. of $9^{\text {th }}$ International Conference on Intelligent Engineering Systems, Mediterranean Sea, Sept. 2005

[11] J N. Sheen, Fuzzy Financial Decision-Making: Load Management Programs Case Study, IEEE Transactions on Power Systems, Vol. 20, No. 4, Nov. 2005, pp. $1808-1817$

[12] M. Stadler, P. Palensky, B. Lorenz, M. Weihs, C. Roesner, Integral Resource Optimisation Networks and their techno-economic Constraints, International Journal of Distributed Energy Resources, Vol. 1, No. 4, 2005, pp. $299-319$

[13] C. Roesner, P. Palensky, M. Weihs, B. Lorenz, M. Stadler, Integral Resource Optimization Network - a new Solution on Power Markets, in proc. of $3^{\text {rd }}$ International Conference on Industrial Informatics, Perth, 2005

[14] P. Palensky, The JEVIS Service Platform - Distributed Energy Data Acquisition and Management, The Industrial Information Technology Handbook, CRC Press, 2005, pp. 111-1 to 111-11

[15] C. Fünfgeld, Repräsentative VDEW-Lastprofile, EUROFORUM Fachkonferenz Netznutzungs- und Bilanzkreismanagement, Berlin, Jul. 2000 